\title{
检出限与灵敏度关系及影响因素的探讨
}

漆红兰*, 李佳妮, 张成孝 ${ }^{*}$

陕西师范大学化学化工学院, 西安 710062

摘要: 检出限和灵敏度是评价分析方法的两个重要指标。国际纯粹与应用化学联合会(IUPAC)等对检出限和灵敏度 的定义给出了明确的推荐。但是, 有些教材和学术论文中检出限和灵敏度的使用仍然较为混乱。本文归纳了检出限 和灵敏度的定义和计算方法, 讨论了现代分析方法检出限和灵敏度的影响因素, 提出了降低检出限和提高灵敏度的 一些策略。

关键词: 检出限; 灵敏度; 标准曲线; 分析化学

中图分类号: G64; O651

\section{Discussion on the Relationship and Influence Factors of the Limit of Detection and the Sensitivity of Analytical Methods}

\author{
Honglan Qi *, Jiani Li, Chengxiao Zhang * \\ School of Chemistry and Chemical Engineering, Shaanxi Normal University, Xi'an 710062, China.
}

\begin{abstract}
The Limit of Detection and Sensitivity of analytical methods are two basic parameters for evaluating analytical methods. The International Union of Pure and Applied Chemistry (IUPAC) clearly recommends their definitions. However, both of them are still somewhat confused in some textbooks and research papers of analytical chemistry. Here, the definitions of both Limit of Detection and Sensitivity are clearly presented and the influence factors on Limit of Detection and Sensitivity of modern analytical methods are discussed. Some strategies to improve the Limit of Detection and the Sensitivity are proposed.
\end{abstract}

Key Words: Limit of Detection; Sensitivity; Standard curve; Analytical chemistry

检出限(Limit of Detection, LOD)和灵敏度(Sensitivity, $S$ ) 是评价分析方法的两个重要指标。国际 纯粹与应用化学联合会(IUPAC) 等对检出限和灵敏度推荐了明确的定义 ${ }^{[1]}$ 。但是, 有些教材和学术论 文中检出限和灵敏度的使用仍然较为混乱。例如, 检出限、检测限、定量限及测定限等均有使用; 一些论文用检出限低表明分析方法的灵敏度高。对检出限和灵敏度及其关系和影响因素已有讨

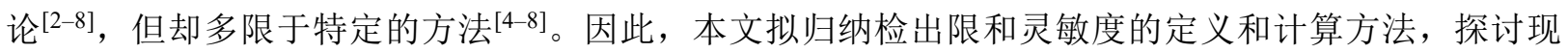
代分析方法检出限和灵敏度的影响因素以及降低检出限和提高灵敏度的一些策略, 抛砖引玉, 共同 商讨, 以准确理解分析方法的检出限和灵敏度的概念和影响因素。

\section{1 检出限和灵敏度的定义与计算}

\section{1 检出限和灵敏度的定义}

1975年, 国际纯粹与应用化学联合会(IUPAC) ${ }^{[1]}$ 对检出限和灵敏度推荐定义为: “The Sensitivity,

收稿: 2021-06-30; 录用: 2021-07-28; 网络发表: 2021-08-19

“通讯作者, Emails: honglanqi@snnu.edu.cn (漆红兰); cxzhang@snnu.edu.cn (张成孝)

基金资助：国家自然科学基金(21775097，21775098)；2017 陕西师范大学名师工作室项目 
$S_{i}$, is defined as the slope of the analytical calibration curve. The Limit of Detection, expressed as the concentration, $c_{L}$, or the quantify, $q_{L}$, is derived from the smallest measure, $x_{L}$, that can be detected with reasonable certainty for a given analytical procedure.” 绝大多数分析化学教材均采用该定义。但是, 在 用词上存在细微差别。例如, 武汉大学主编的《分析化学》 (第五版) ${ }^{[9]}$ 中指出 “仪器或分析方法灵敏 度是区别具有微小浓度差异分析物能力的度量; 检出限(detection limit)又称检出下限或最低检出量 等, 定义为一定置信水平下检出分析物或组分的最小量或最低浓度。” 华中师范大学等主编的《分析 化学》 (第四版) ${ }^{[10]}$ 中指出 “被测物质单位浓度或单位质量的变化引起响应信号值变化的程度, 称之 为方法的灵敏度(sensitivity); 某一方法在给定置信水平上能够检出被测物质的最小浓度或最小质量 称为这种方法对该物质的检出限(detection limit)。” 全国科学技术名词审定委员会(原称 “全国自然科 学名词审定委员会” )发行的《化学名词》(第二版) ${ }^{[11]}$ 推荐: “灵敏度是被测组分的量或浓度改变1个 单位时分析信号的变化量。” 进一步指出 “在仪器分析中, 分析灵敏度直接依赖于检测器的灵敏度与 仪器的放大倍数。由于灵敏度未能考虑到测量噪声的影响, 现在已不用灵敏度来表征分析方法的最 大检出能力, 而推荐用检出限来表征。”

在建立一种新分析方法或实际测定时, 通常需要绘制一个分析信号 $y$ (纵坐标) 随标准溶液的浓度 $c$ 或质量 $q$ (横坐标)的变化曲线, 称为标准曲线。在标准曲线上有一段线性部分, 灵敏度即为 “标准 曲线线性部分的斜率”, 用线性部分对应的 $\Delta y / \Delta c$ (或 $\Delta y / \Delta q$ ) 来表示灵敏度 $S$ 。

检出限为使用统计学方法判断信号能显著区别于空白信号的最小信号值对应的最小浓度(或最 小质量)。用更容易理解的一句话说, 检出限就是某方法有一定的把握估计能检出某组分的最小浓度 (或最小质量)。

\section{2 检出限的计算}

根据IUPAC推荐LOD的定义 ${ }^{[1]}$, 最小可鉴别的检测信号 $y_{\min }$, 即为比空白信号 $\bar{y}_{\mathrm{b}}$ 有一定程度 $k s_{\mathrm{b}}$ 区 分的信号:

$$
y_{\min }=\bar{y}_{\mathrm{b}}+k \mathrm{~s}_{\mathrm{b}}
$$

用浓度或质量分别定义LOD为:

$$
\begin{aligned}
& \operatorname{LOD}_{\mathrm{c}}=\left(y_{\min }-\bar{y}_{\mathrm{b}}\right) / S_{\mathrm{c}} \\
& \operatorname{LOD}_{\mathrm{q}}=\left(y_{\text {min }}-\bar{y}_{\mathrm{b}}\right) / S_{\mathrm{q}}
\end{aligned}
$$

则LOD的计算式为:

$$
\begin{aligned}
& \operatorname{LOD}_{\mathrm{c}}=k \cdot s_{\mathrm{b}} / S_{\mathrm{c}} \\
& \operatorname{LOD}_{\mathrm{q}}=k \cdot s_{\mathrm{b}} / S_{\mathrm{q}}
\end{aligned}
$$

其中, $\bar{y}_{\mathrm{b}} 、 s_{\mathrm{b}}$ 分别为 $n$ 次测定空白得到的信号平均值和标准偏差; $k$ 为在所选置信度下对应的数值 因子, 也称置信因子; $S_{\mathrm{c}}$ 和 $S_{\mathrm{q}}$ 分别为以浓度和质量表示的灵敏度, 即 $S_{\mathrm{c}}=\Delta y / \Delta c, S_{\mathrm{q}}=\Delta y / \Delta q$ 。

笔者将对检出限的计算参数进行进一步的讨论。

\subsection{1 测量次数 $n$}

测量数据一般服从正态分布规律。IUPAC推荐: 空白样品重复测定次数应足够多, 应大于 20 次,

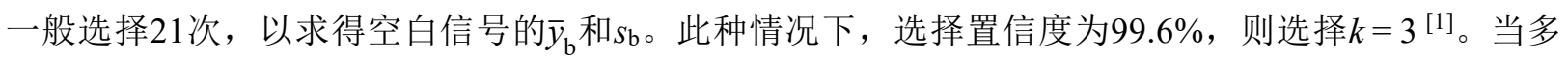
个实验室同时评价某个标准分析方法时, 选择 $n=21$; 当评价建立的某种新方法时, 一般选择 $n=11$ 或7。

\subsection{2 置信因子 $k$}

置信因子 $k$ 的选择与数据服从的分布规律和置信度的确定相关。当测量数据服从正态分布时, $k=3$ 对应的置信度为 $99.6 \%$; 而当测量次数有限且数据不符合正态分布时, $k=3$ 对应的置信度大约 为 $90 \%{ }^{[1]}$ 。

如果测量数据服从正态分布, 也可以从统计学假设检验理解检出限。如图1所示 ${ }^{[12]}$, 依据Currie 建议 ${ }^{[13]}$, 先确定一个临界值(Critical value, $L_{\mathrm{C}}$ ), 在该值的基础上再确定检出限(Detection limit, $L_{\mathrm{D}}$ )。 
临界值是人为设定的判断待测组分是否存在的标准, 检出限是实际中能可靠检出的最小信号或最小 浓度。假设低浓度范围内测量信号服从正态分布且标准偏差为常数, 设定 $\alpha$ ( “实际不存在但误判为 存在” 的概率 $)=\beta$ ( “实际存在但误判为不存在” 的概率 $)=5 \%$, 则 $k=3.29^{[13]}$ 。

如果测量数据服从 $t$ 分布, 即少量测量次数数据的分布规律, 我们可以从统计学假设检验理解最 小可鉴别的检测信号 $y_{\min }$ 与置信因子 $k$ 、置信度 $P$ 和平行测定次数 $n$ 之间的关系, 进而深入理解检出限。 如果假设 $k=t_{\mathrm{P}, \mathrm{f}} / n^{1 / 2}$, 式 1 可转换为式 $6:$

$$
y_{\min }=\bar{y}_{\mathrm{b}}+s_{\mathrm{b}} \frac{t_{\mathrm{P}, \mathrm{f}}}{n^{1 / 2}}
$$

$t_{\mathrm{P}, \mathrm{f}}$ 为统计量 $t_{\mathrm{P}, \mathrm{f}}$ 值表 (双边) ${ }^{[10]}$ 查得的数值, $f=n-1$ 。由式 6 可知, 在空白信号和空白信号标准 偏差一定的情况下, $t_{\mathrm{P}, \mathrm{f}} / n^{1 / 2}$ 值越大, 则检出限越高。一般来说, 在测定次数 $n$ 相同条件下, $P$ 越高, $t_{\mathrm{P}, \mathrm{f}}$ 就越大, 则 $t_{\mathrm{P}, \mathrm{f}} / n^{1 / 2}$ 值就越大。例如: $t_{90 \%, 2}=2.92$, 则 $t_{\mathrm{P}, \mathrm{f}} / n^{1 / 2}=1.68 ; t_{95 \%, 2}=4.30$, 则 $t_{\mathrm{P}, \mathrm{f}} / n^{1 / 2}=2.48$;

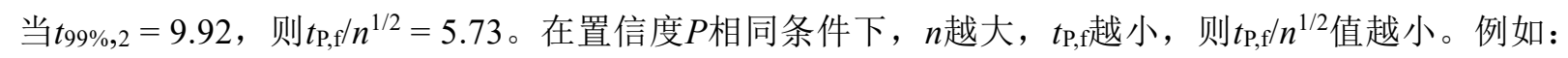
$t_{99 \%, 2}=9.92$, 则 $t_{\mathrm{P}, \mathrm{f}} / n^{1 / 2}=5.73$; 当 $t_{99 \%, 4}=4.60$, 则 $t_{\mathrm{P}, \mathrm{f}} / n^{1 / 2}=2.06$; 当 $t_{99 \%, 6}=3.71$, 则 $t_{\mathrm{P}, \mathrm{f}} / n^{1 / 2}=1.42$ 。在 一般评价新方法时, 通常选择 $k=3$ 。
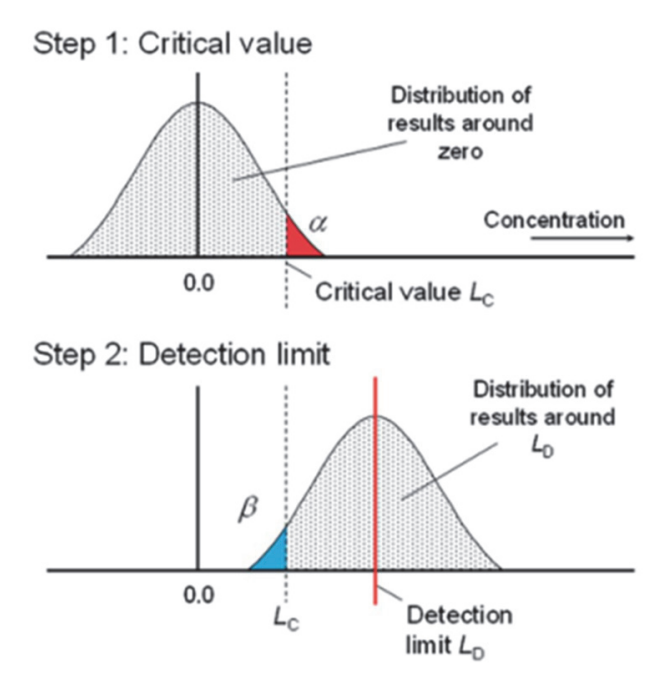

图1 假设检验法确定检出限 ${ }^{[12]}$

\subsection{3 灵敏度 $S$}

在化学测量中, 测量所获信号 $y$ 与浓度 $c$ 的关系即标准曲线一般呈 S型, 如图2所示。为了便于理 解, 在该曲线分别标注 $A 、 B 、 C 、 D 、 E$ 点, 即标准曲线由 $A B 、 B C 、 C D 、 D E$ 四段组成。LOD介于 $A B$ 段; $\mathrm{CD}$ 段为一般所称的线性范围。如果用 $S_{1} 、 S_{2}$ 分别表示 $\mathrm{BC} 、 \mathrm{CD}$ 段的斜率。从式 4 可知, $k$ 和 $s_{\mathrm{b}}$ 一定 的情况下, $S_{\mathrm{c}}$ 越大, 则 $\mathrm{LOD}_{\mathrm{c}}$ 就越小。由式1可知, $y_{\min }$ 为空白信号平均值与 $k s_{\mathrm{b}}$ 之和。从图 2 左下角可 以看出, $y_{\min }$ 与 $S_{2}$ 对应的 $\mathrm{LOD}_{2}$ 和 $y_{\min }$ 与 $S_{1}$ 对应的 $\mathrm{LOD}_{1}$, 因为 $S_{2}>S_{1}$, 则 $\mathrm{LOD}_{2}<\mathrm{LOD}_{1}$ 。有些人用检出 限低表示灵敏度高, 这并不一定正确, 因为检出限还与空白值的标准偏差相关。一般认为LOD为线 性范围下限的 $1 / 2-1 / 3$ 比较可信。

若测量信号与浓度的对数 $\lg c$ 成正比, 此时检出限又该如何计算呢? 电位分析法就是一个典型的 例子。Dillingham等人提出了一个与IUPAC推荐相一致的检出限计算方法 ${ }^{[14]}$ 。他们使用离子选择性电 极的电位方程, $E=E^{\ominus}+\beta_{1} \log \left(a+\beta_{2}\right)+\varepsilon$, 推导出计算离子选择性电极法的LOD计算式为:

$$
\operatorname{LOD}_{\alpha, \beta}=\beta_{2}\left(10 \frac{\left(z_{\alpha+z \beta}\right) \sigma}{\left|\beta_{1}\right|}-1\right)
$$

式7中, $\beta_{1}$ 为与温度及待测离子电荷数有关的斜率参数, $\beta_{2}$ 为与干扰离子的活度、选择性、化合价有 
关的参数, $\sigma$ 是空白信号的标准偏差, $a$ 和 $\beta$ 为假阳性率和假阴性率, $z_{\alpha}, z_{\beta}$ 分别为与 $a$ 和 $\beta$ 相关的标准 正态分布的参数, 即为与置信度相关的置信因子。

对于某种方法的线性回归方程服从 $y=a+S \lg c$ 的情况 ( $a$ 为截距), 按照 $\log c=\left(y_{\min }-\bar{y}_{\mathrm{b}}\right) / S=3 s_{\mathrm{b}} / S$, 可推导出计算检出限的公式为:

$$
\mathrm{LOD}=10^{3 s_{\mathrm{b}} / S}
$$

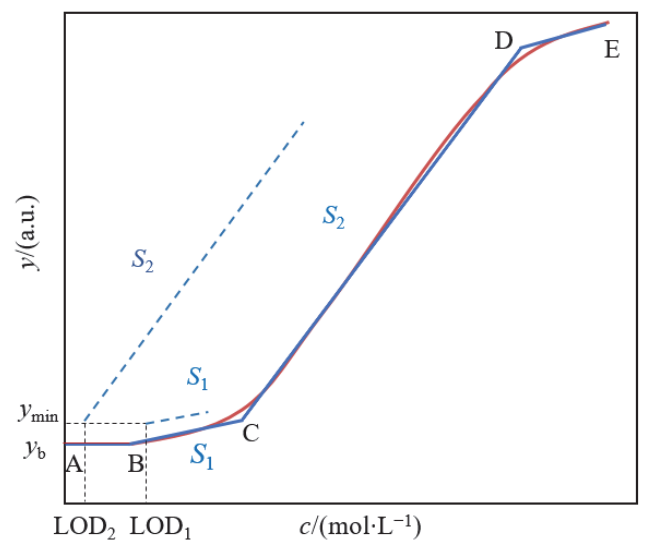

图2 标准曲线应用于剖析检出限和灵敏度关系的示意图

\section{2 检出限、定量限及测定限的区别}

本文拟就分析化学中几个中文名词与英文名词物理含义给予解释。全国自然科学名词审定委员

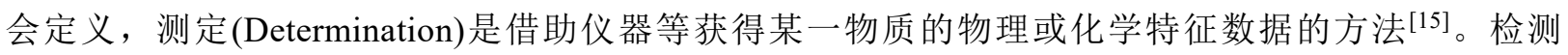

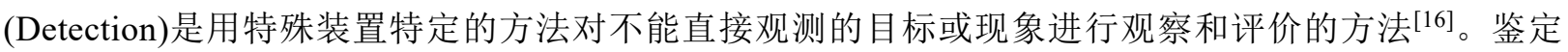
(Identification) 是对试样中某种组分的鉴别和确定的过程 ${ }^{[11]}$ 。测量(Measurement) 是通过实验获得并可 合理赋予某量一个或多个量值的过程 ${ }^{[17]}$ 。笔者认为, 测定(Determination)侧重表达定量分析的概念; 定量(Quantification)也侧重表达定量分析的概念; 检测(Detection)侧重表达定性分析的概念; 鉴定 (Identification)侧重表达识别的概念; 测量(Measurement)是按照某种规律, 用数据来描述观察到的现 象, 即对事物做出量化描述。

定量限(Limit of Quantification, LOQ)与检出限(LOD)为两个不同的参数。全国自然科学名词审 定委员会发行的《化学名词》(第二版)推荐 ${ }^{[11]}$, 定量限为 “根据统计学原理给出的用于估算能定量测 定分析物的最小含量或浓度。若样品中存在的分析物的量大于该量值, 则可认为该样品可以某一相 对标准偏差被定量测定。在误差分布遵从正态分布的条件下, 以适当的置信概率(95\%)被定量测定的 最小含量或浓度。” Currie建议 ${ }^{[13]}$, “Quantification limits are defined in terms of a specified value for the relative standard deviation”。美国化学会环境改善委员会 (ACS Committee On Environmental Improvement) 建议 ${ }^{[18],} \mathrm{LOD}=S_{b}+3 \sigma, \mathrm{LOQ}=S_{b}+10 \sigma$, 其中 $S_{b}$ 是空白信号测量的平均值, $\sigma$ 是空白 信号测量的标准偏差。图3将浓度划分为三个区域, 理解LOD和LOQ的区别。若实际浓度小于LOD, 则认为 “未检出”; 若实际浓度在LOD-LOQ之间, 则认为可 “定性检测”; 若实际浓度大于LOQ, 则 认为可 “定量测定”。

测定限(Limit of Determination, Determination Limit)为定量分析实际可能测定的某组分的下 限 ${ }^{[11]}$ 。因此, 检出限与定量限和测定限为三个不同的参数。

\section{3 影响灵敏度的因素和提高灵敏度的策略}

如前所述, 灵敏度为单位浓度(或质量)变化而引起的测量信号的变化 $\left(S_{\mathrm{c}}=\Delta y / \Delta c, S_{\mathrm{q}}=\Delta y / \Delta q\right)$ 。 对于信号增强法, 其值为正; 对于抑制法, 其值为负。测定方法的灵敏度主要取决于测定体系物质 


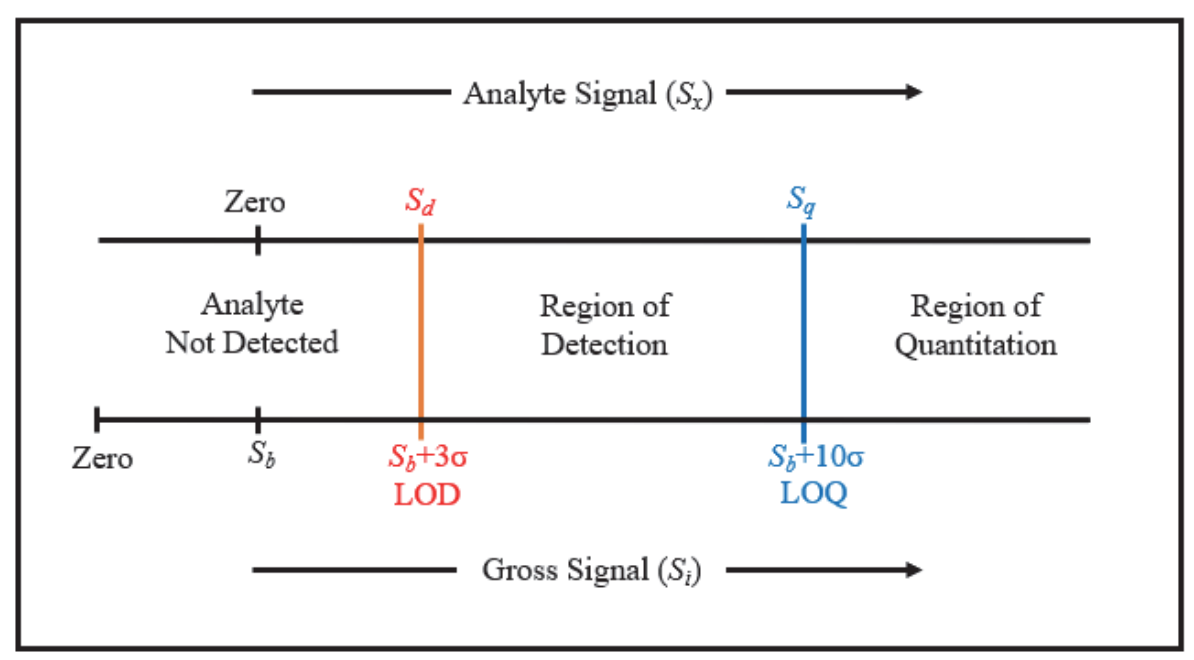

图3 检出限、定量限将浓度分为三个区域[18]

的性质(包括溶液条件)、测定仪器参数和所用仪器设备等因素。

对于给定的化学和生物分析方法, 测定物质转化为测量信号是关键因素。分析方法可分为直接 法和间接法。直接法是直接利用待测物质产生的测量信号进行测量的方法; 间接法是将待测物质转 化为可产生测量信号的物质进行测量的方法。以下影响因素和策略是针对产生测量信号的物质进行 讨论的。

1) 光度定量分析法

其定量公式为: $A=\log \frac{I_{0}}{I}=\varepsilon b c$ 。 $\varepsilon b$ 即方法的灵敏度 $S$, 增大摩尔吸光系数 $\varepsilon$ 和光程长度 $b$ 均可提高 方法灵敏度。增大吸光分子横截面积、选择待测组分最大吸收波长为测量波长、选择适宜的溶剂、 $\mathrm{pH}$ 等, 有可能增大 $\varepsilon$; 增加光程 $b$, 也可增大 $S$, 但是 $b$ 太大 $(>2 \mathrm{~cm})$ 会造成光损失而使实际的入射光强 度 $I_{0}$ 降低。

\section{2) 苂光定量分析法}

其定量公式为: $I_{\mathrm{f}}=2.3 k \varphi_{\mathrm{f}} I_{0} \varepsilon b c$ 。 $2.3 k \varphi_{\mathrm{f}} I_{0} \varepsilon b$ 即方法的灵敏度 $S$ 。选择苂光量子产率 $\varphi_{\mathrm{f}}$ 大和 $\varepsilon$ 大的物 质是提高 $S$ 的关键; 选择入射光强度 $I_{0}$ 大和仪器参数 $k$ 大的仪器和 $b$ 大的测量池, 也可提高 $S$ 。使用激光 光源可提高 $I_{0}$, 也允许使用较大的 $b$ 。好的苂光分光光度计有较好仪器放大因子 $k$, 也常受到青睐。

3) 伏安分析法

对于平面电极，线性扫描扩散电流定量公式为Randles-Sevcik方程： $i_{\mathrm{p}}=2.69 \times 10^{5} n^{3 / 2} D_{\mathrm{o}}{ }^{1 / 2} A v^{1 / 2} c$ 。 其中 $i_{\mathrm{p}}$ 为峰电流 $(\mathrm{A}), n$ 为转移电子数, $D_{\mathrm{o}}$ 为扩散系数 $\left(\mathrm{cm}^{2} \cdot \mathrm{s}^{-1}\right), A$ 为电极面积 $\left(\mathrm{cm}^{2}\right), v$ 为扫描速率 $\left(\mathrm{V} \cdot \mathrm{s}^{-1}\right)$, $c$ 为待测组分浓度 $\left(\mathrm{mol} \cdot \mathrm{cm}^{-3}\right.$, 或 $\left.\mathrm{mol} \cdot \mathrm{mL}^{-1}\right) 。 2.69 \times 10^{5} n^{3 / 2} D_{\mathrm{o}}{ }^{1 / 2} A v^{1 / 2}$ 即方法的灵敏度 $S$ 。由此可见, 增大 电极面积 $A$, 增大扫描速率 $v$, 增大扩散系数 $D$ 等, 则可增加峰电流。但是, 应注意测得的峰电流为残 余电流和扩散电流之和。残余电流主要包括杂质电流和充电电流 $i_{\mathrm{c}}$ 。充电电流 $i_{\mathrm{c}}=A C_{\mathrm{d}} v$, 式中 $C_{\mathrm{d}}$ 为电 极的微分电容。由此可见, 增大扫描速度, 充电电流的增加比扩散电流的增加更快, 不利于检出限 的降低; 但形式上增加了方法的灵敏度。

对于分析化学研究者, 对于给定的体系, 常常研究化学或生物信号放大策略以提高方法的灵敏 度。例如, 酶催化放大策略是利用检测物的循环利用进行物质放大的策略; 有些纳米信号放大策略 是利用纳米材料对信号物质的多负载提高测量信号的策略; 生物信号放大策略如PCR 是进行检测 基因片段物质放大的策略。又如, 对于伏安法测定方法尽量设计成基于测量平行催化电流的方法, $i_{\mathrm{ca}}=n F A D^{1 / 2}\left(k_{\mathrm{f}} c_{\mathrm{z}}\right)^{1 / 2} c$, 式中 $k_{\mathrm{f}}$ 为均相反应速率常数, $c_{\mathrm{z}}$ 为氧化剂(或还原剂)浓度, $c$ 为待测物(催化剂) 浓度。相对于扩散电流体系或 $k_{\mathrm{f}}$ 小的催化体系, 选择 $k_{\mathrm{f}}$ 大的体系就可以提高灵敏度。 


\section{4 影响检出限的因素和降低检出限的策略}

从式4可知, 检出限与方法灵敏度、重现性、置信因子的选取等因素有关。提高方法的灵敏度和 降低空白信号的标准偏差, 即可降低检出限。从式1-式 3 可知, 检出限也与空白信号 $\bar{y}_{\mathrm{b}}$ 有关; 但是, 在式4中并没有体现出 $\bar{y}_{\mathrm{b}}$ 。尽管在实际工作中, 对于高 $\bar{y}_{\mathrm{b}}$ 可以通过仪器方法进行抑制或采用数据处理 进行部分扣除。然而, 高背景噪声必定会对分析方法的实际性能指标造成负面的影响。在此, 主要 讨论降低检出限的策略。

背景噪声主要来源于仪器背景、化学背景和生物背景噪声 ${ }^{[19,20]}$ 。仪器背景噪声主要来源于电子 元件的噪声, 如光电倍增管的暗电流, 可选择暗电流小的光电倍增管; 如温度变化引起电子元件信 号的波动, 可选择恒温体系减小此种波动; 又如伏安分析中外电场或磁场引起的电流波动, 可选择 法拉第笼减小外电场的干扰。化学背景即为杂质或未知物质产生的微小信号。可通过提纯试剂和溶 剂来减小这类背景, 也可以加入大量某种物质使化学背景恒定而进行扣除。在实际工作中, 样品中 常常存在干扰物质, 干扰物质产生的负干扰或正干扰并不属于此类。生物背景主要来源于检测生物 体系(组织、细胞等)中未知物质产生的微小信号。对于这类背景应选择标准的组织或细胞等, 一般使 产生的生物背景尽量恒定, 再进行扣除。

上述重点讨论了与标准曲线相关的灵敏度和检出限。在实际工作中, 常常需要绘制工作曲线进 行定量分析。即根据实际样品, 并考虑消除各种干扰而加入合适的试剂或适当地改变仪器测量参数 绘制工作曲线。近年来, 关于生物分析和生物传感器中灵敏度和检出限的讨论备受关注 ${ }^{[19-21]}$ 。在生 物分析中, 生物背景对分析结果影响最大。当生物传感器检测实际生物样品时, 样品基体中的生物 大分子会干扰待测组分与传感器之间的相互作用, 导致传感器的性能变差。例如, 毛兰群 ${ }^{[19]}$ 指出, 植入式传感器在活体检测一定时间后, 由于周围生物层的形成以及体内的免疫反应, 会影响其分析 性能, 甚至出现信号异常和传感器故障。在实际样品中计算获得待测物质的检出限可能高于标准溶 液求得的检出限。复杂生物样品中的分析结果有时也会优于标准溶液中的分析结果, 例如, 血样中 抗原与抗体的结合能力大于缓冲溶液中两者的结合能力, 在血样中分析有利于免疫分析方法分析性 能的提高 ${ }^{[20]}$ 。因此, 对于新建立的某一新分析方法的性能评价, 应报告该方法在标准溶液中以及在 真实的样品中的灵敏度和检出限。因为后者更切合实际分析。

本文剖析了灵敏度和检出限的经典定义及计算公式, 讨论了影响方法灵敏度和检出限的因素, 对提高灵敏度、降低检出限提出了一些策略。总体而言, 分析方法的灵敏度和检出限, 两者相辅相 成, 不能孤立使用。本文旨在抛砖引玉, 引起大家对方法灵敏度和检出限的概念及影响因素的关注 与思考，共同商讨，以促进两者的规范使用。

\section{参 考 文 献}

[1] IUPAC. Pure Appl. Chem. 1976, 45, 101.

[2] 杨淳, 郑姗, 刘洋, 施娅颖, 黄朝表. 大学化学, 2018, 33 (5), 51 .

[3] 察冬梅. 大学化学. 2011, 26 (4), 84.

[4] Ingle, J. D. J. Chem. Educ. 1974, 51, 100.

[5] Foley, J. P.; Dorsey, J. G. Chromatographia 1984, 18, 503

[6] Desimoni, E.; Brunetti, B. Electroanalysis 2013, 25, 1645.

[7] 文慧, 刘珣, 张尚青, 杨婷, 陈明丽, 王建华. 分析化学, 2019, 47 (4), 597.

[8] Easterling, L. F.; Yerabolu, R.; Kumar, R.; Alzarieni, K. Z.; Kenttämaa, H. I. Anal. Chem. 2020, 92, 7471.

[9] 武汉大学. 分析化学(下册). 第5 版. 北京: 高等教育出版社, 2007.

[10] 华中师范大学, 等. 分析化学(下册). 第4版. 北京: 高等教育出版社, 2012 .

[11] 全国自然科学名词审定委员会. 化学名词. 第2版. 北京: 科学出版社, 2016. 
[12] Analytical Methods Committee AMCTB No. 92. Anal. Methods 2020, 12, 401.

[13] Currie, L. A. Pure Appl. Chem. 1995, 67, 1699.

[14] Dillingham, P. W.; Alsaedi, B. S. O.; Granados-Focil, S.; Radu, A.; McGraw, C. M. ACS Sensors 2020, 5, 250.

[15] 全国自然科学名词审定委员会. 化工名词(三)化学工程基础分册. 北京: 科学出版社, 2019.

[16] 全国自然科学名词审定委员会. 核医学名词. 北京: 科学出版社, 2018 .

[17] 全国自然科学名词审定委员会. 计量学名词. 北京: 科学出版社, 2015.

[18] ACS committee on environmental improvement. Anal. Chem. 1980, 52, 2242.

[19] Mao, L. ACS Sensors 2020, 5, 2659.

[20] Masson, J. F. ACS Sensors 2020, 5, 3290.

[21] 张秀明, 冯仁丰. 中华检验医学杂志, 2014, 37 (9), 669. 